\title{
A Real Time Vein Detection System
}

\author{
Kazi Istiaque Ahmed, Mohamed Hadi Habaebi, Md Rafiqul Islam \\ Department of Electrical and Computer Engineering, Kulliyyah of Engineering, International Islamic University \\ Malaysia
}

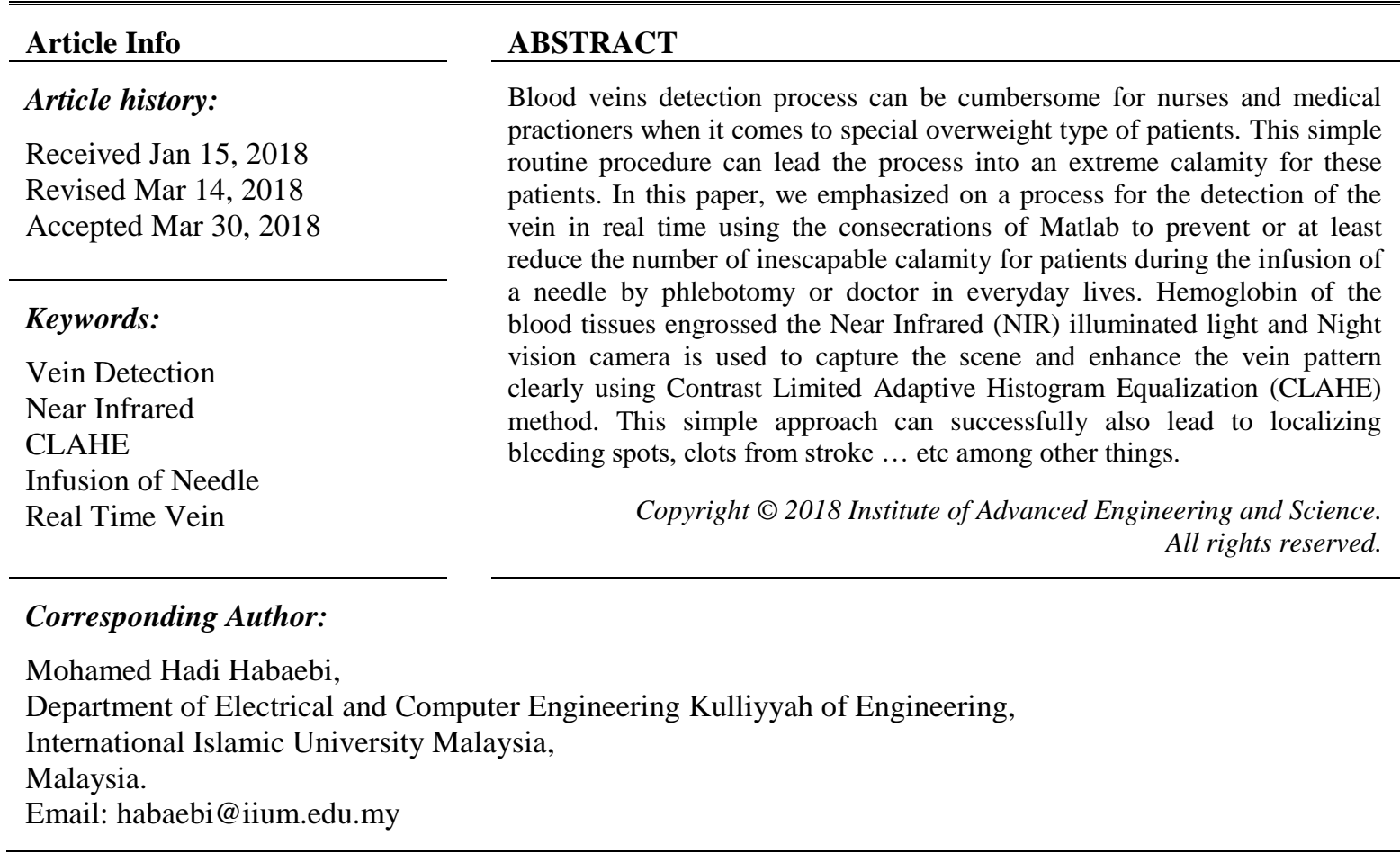

\section{INTRODUCTION}

Nowadays, the infusion of a needle to the patients for hospitalization has been done by different medical operators in the field of medicine and the Operators also require to find out the veins for the donation of blood, transfusion of blood and injection of the ampule liquid or fluid into the body [1]. It is a very important process for the phlebotomist or the clinician to find the vein vessel to avoid needless pain due to the repeated skin at poking of the patient. For locating the vein in the hand of the patients, many of them face the difficulties during the period of insertion of a needle in the first time, which can create a panic atmosphere for the patients and, as a result, the process will take longer time. Furthermore, imaging utilizing Near Infrared (NIR) has turned out to be an exceptional instrument in restorative diagnostics. For example, NIR is used in Optical Intelligibility Tomography equipped for 3D imaging with high determination keeping pace with low-control microscopy, in measuring bloodstream, blood volume, $\mathrm{O} 2$ utilization, re-oxygenation rates and muscle recuperation time in muscle, in the noninvasive evaluation of cerebrum work and other medical applications [2], [3]. A standard lens camera cannot view the NIR images because it requires an IR none-blocking filter to allow it to view this range from the electromagnetic spectrum. Most shocking information is that, the rate of failure in needle infusion is approximately $3-5 \%$ in the first endeavor among 500 milliard on the human physique for different purpose, [4] quantify this by their exploration.

The patterns of the veins are core characters and the skin is the protector of the veins which are not exaggerated by bricklaying, propagation, scratches, and wounds etc. kind of activities. Vein location is the technique for dissecting the vein designs or the examples of veins under the skin and it is conceivable to distinguish the veins from the binary frame from erratically begin position utilizing line tracking technique [5]. However, the radiation of infrared is emitted by humans and the term thermography can be defined as the portrait produced by an infrared camera where it does not require light and is not affected by the variation in the illumination [6]. Far-infrared (FIR) captures the tissue having a higher temperature than the adjacent 
tissue in the surface and Near-infrared (NIR) captures the blood venous engrosses maximum of the infrared radiation than the adjacent tissue. Moreover, the NIR and FIR both cameras captured the light beyond the view of humans and the range of NIR illumination is around 740 nanometers to 940 nanometers and is capable to infiltrate $5 \mathrm{~mm}$ subterranean into the skin tissue [7], [8].

Vein Viewer and CMOS camera and XBee pair with IR illuminator is a line of product which gives the practitioner a real-time image projected in on the patient arm and it uses the principle that infrared light is absorbed by the blood more than the surrounding tissue [3], [7]. However one of the most difficult issues in vein shape distinguishing proof by utilizing sensor combination between the devices [9]. Moreover, vein detection can be drastically enhanced by modifying camera lens to IR sensitivity through removing IR filter with respect to RGB camera [10]. However, all the modern camera lenses have IR-CUT Filter in the sensor area due to focusing on the visible lights only [11].

The color of objects is determined by the percentage of absorption and reflection of different wavelengths projected toward that object, we can see red a ball red because it absorbs the wavelengths of blue and green while reflecting red. The reflection and absorption of wavelengths are limited to the visible light as the rest of the wavelength spectrum also follow the same thing [12]. Contrariwise, the fascinating thing is that the band close to the red wavelength the near infrared range the blood react in totality different way where it absorbs it [13].

Moreover, the human eyes only able to see in the visible wavelength between $380 \mathrm{~nm}$ which treated as violet and 720nm which red and The tissues underlying in the human skin absorbs the light differentially where the absorption ratio can be increased by the deoxygenated blood hemoglobin [14]. However, the vein cannot be seen clearly on the skin [15] because of its diverted skin tone [7].

On the other hand, there are different technique can be considered to be used for the enhancement of images but the critical work to get the clearer view of the vein from the human body parts in real-time. Moreover, the in Bi-Histogram Equalization (BBHE) procedure the images are extracted to create multiple sub-image and between two consecutive part of images the average to preserve the average brightness of the input image are providing as an output [16]. The author illustrates a comparison of the output results among BBHE, RMSHE \& Recursive Sub Image Histogram Equalization (RSIHE) over a gray image and found impressive output for RSIHE but the recursively separation on the histogram. Nevertheless, in the Recursive Mean Separate Histogram Equalization (RMSHE) these sub-images of the input image is done through the equalization of the inputs recursion level [17], [18]. Contrariwise, from [19], the tabulated information at Table 1 shows that the CLAHE is extremely exceptional over all the procedure for the vein images.

Our aim in real time Thermography where the NIR area of the electromagnetic spectrum is used to measure the heat transmitted or reflected from the human body or to create an image that allows us to detect the veins near the skin surface and we can determine the vein from the frame and enhance the pattern of the vein by Matlab. This rest of the paper is structured as follows. Section 2 discusses related works and the theory of human skin interaction with NIR and the contrast limited adaptive histogram equalization CLAHE algorithm, section 3, introduces the proposed system for designing a real-time vein detection, section 4, sum up results and analysis and section 5, concludes the paper. 
Table 1. The histogram comparison among dissimilar image enhancement technique and their output
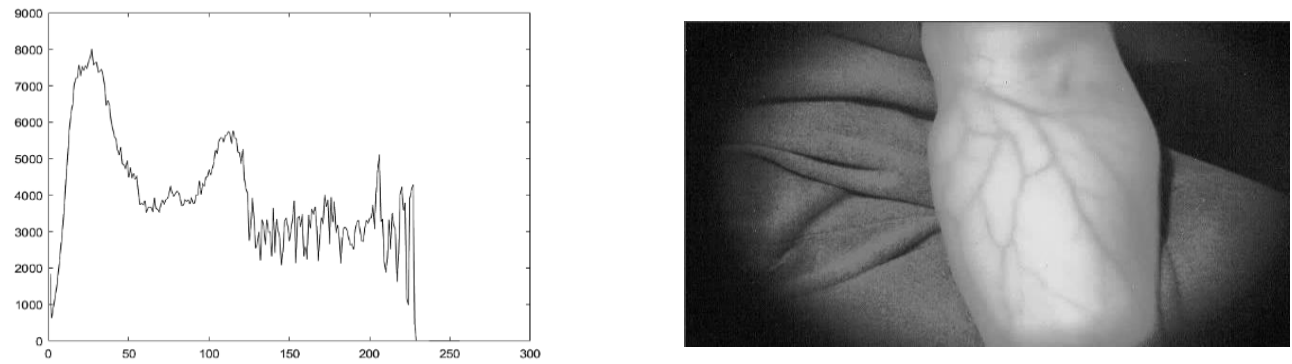

(a)

with Entropy 7.7316
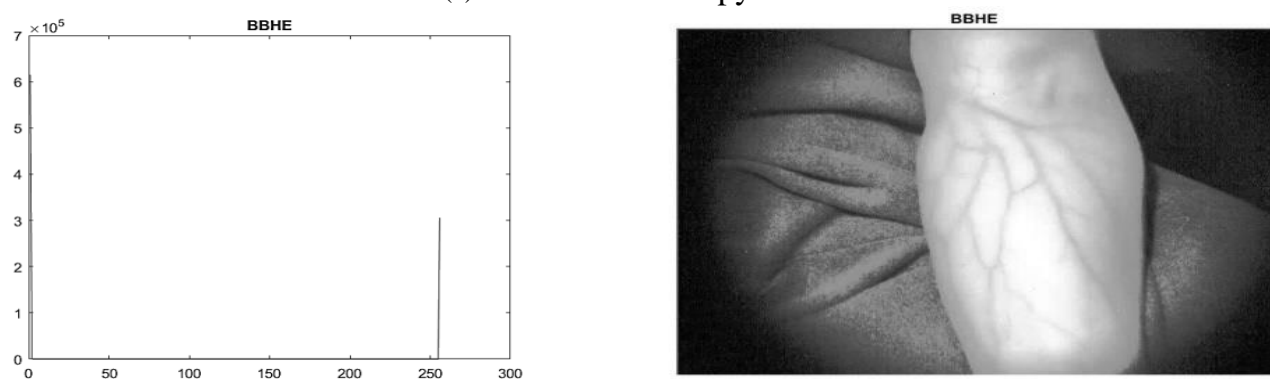

(b)

with Entropy 0.91763 \& MSE 238.6895 and PSNR 24.3525
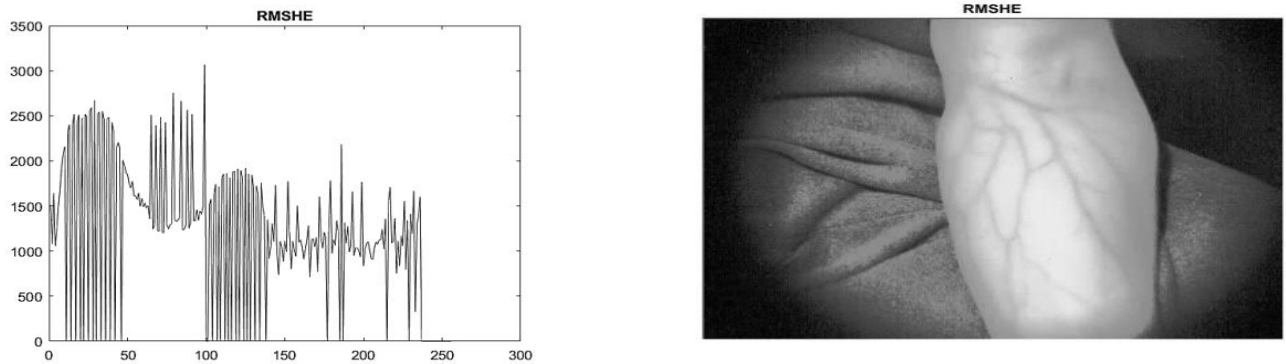

(a)

with Entropy 7.5759 \& MSE 70.2982 and PSNR 29.6614
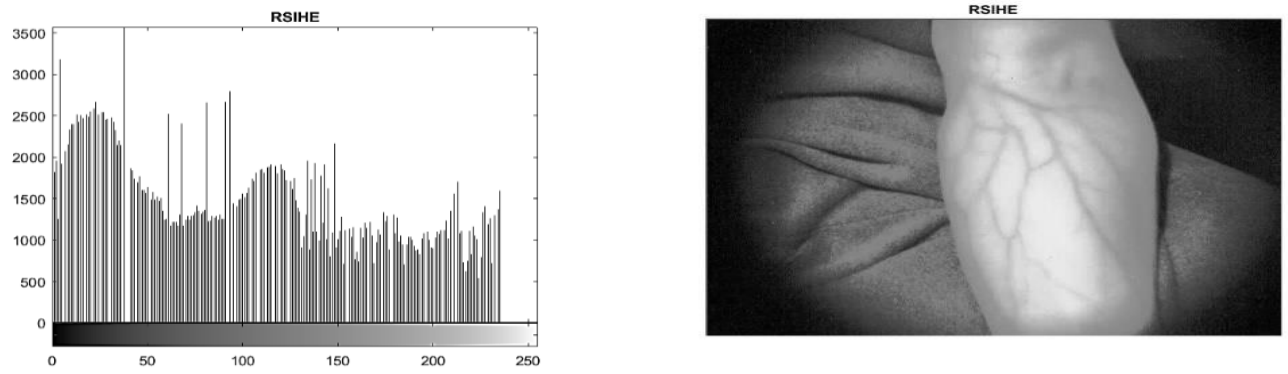

(a)

with Entropy 7.5932 \& MSE 17.3980 and PSNR 35.7258
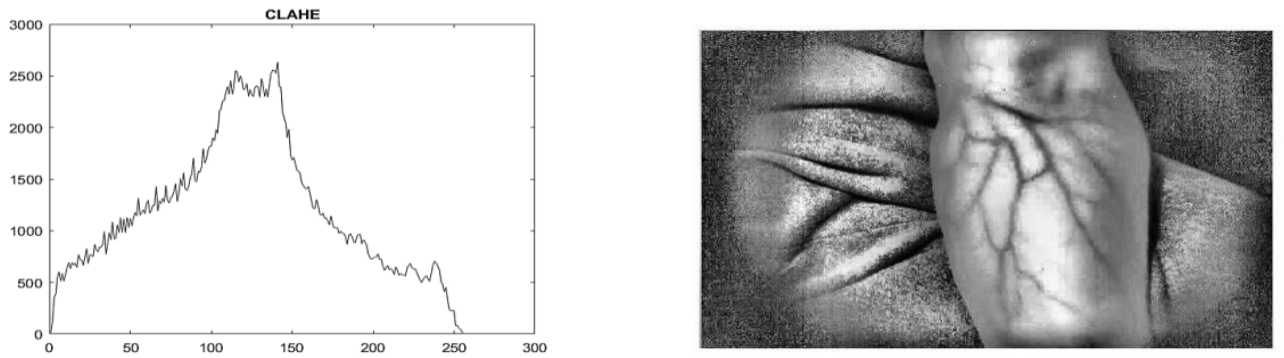

(e) with Entropy 7.7798 with Entropy 7.5932 \& MSE 2693.8455 and PSNR 13.8271 


\section{RESEARCH METHOD}

\subsection{NIR Imaging and Human Skin}

NIR illumination is an imaging technique to view between wavelengths of $720 \mathrm{~nm}$ and $1000 \mathrm{~nm}$ where human eye and general purpose camera unable to detect as the Figure 1 showing all visible and invisible wavelengths. The main purpose of these techniques is used to view more deeply in an image without ignoring the Infrared wavelength. In general imaging techniques, the invisible spectrum is ignored and represented as color correction or false color of the image for better presentation in a human eye.

\section{LIGHT SPECTRUM}

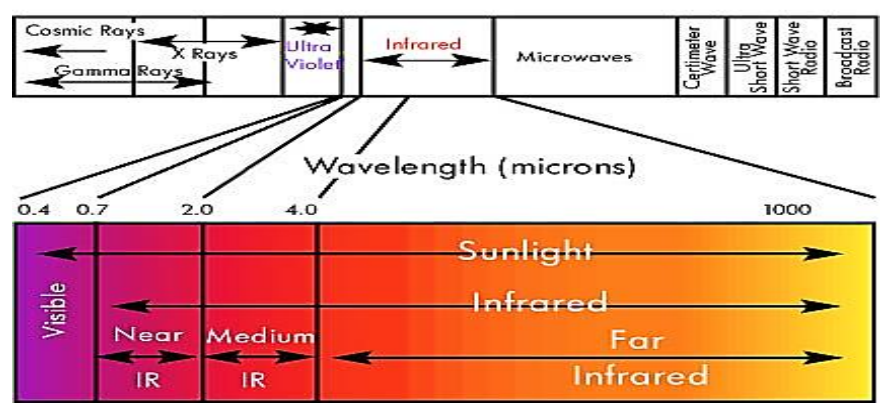

Figure 1. Near Infrared (NIR) Light Spectrum [20]

The human skin can be divided into two layers the epidermis and the dermis, below there is a subcutaneous fat layer, depending on the concentration of melanin, blood, and keratin the reaction may vary. In the therapeutic review point lights in wavelengths in the vicinity of $720 \mathrm{~nm}$ and $950 \mathrm{~nm}$, infiltrates profoundly into tissues, organically took into consideration non-intrusive examination. In this way, the wavelength of the infrared light bar turning out from a light source is chosen to be inside the close infrared district with a common wavelength around $850 \mathrm{~nm}$. Utilizing this wavelength, as Figure 2, it likewise maintains a strategic distance from unwanted impedance from the IR radiation produced by the human body and the earth.

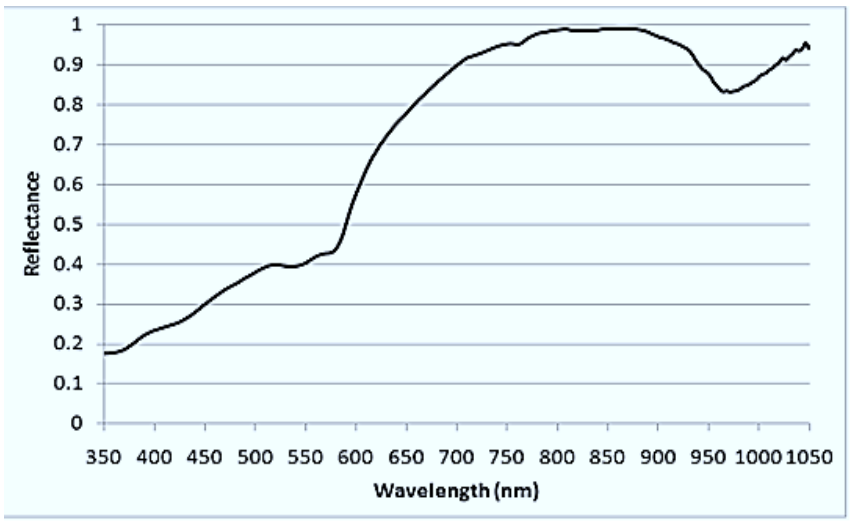

Figure 2. Human skin infrared reflection [12]

Moreover, the vein pictures by the assistance of NIR LED is noninvasive with no known unsafe impacts. It isn't trustworthy for distinguishing profound supply routes and veins, for example, the advanced conduit [14].

\subsection{Contrast Limited Adaptive Histogram Equalization (CLAHE)}

The CLAHE is an algorithm which was developed in 1994. The primary thought of this calculation is to cut the produced histogram in the dark level incline of a picture where the pixel in each area is similarly 
appropriated with the privately created histogram and it is trailed by redistributing of the pixels over the entire histogram to influence the histogram to process more indistinguishable [14].

The difference factor is characterized as exclusive of the normal histogram substance in a low factor neighborhood histograms, the most extreme incline will be low and in this way result in restricted differentiation improvement. In spite of the fact that the picture created by CLAHE prepared to utilize a high differentiation factor, picture clamor is as yet worthy. The primary favorable circumstances of the CLAHE change are the humble computational prerequisites, its usability, and its exclusive outcomes on general pictures which can be Applied only to that category of images [21] where different normal Adaptive Histogram Enhancement (AHE) techniques are not suitable. The main signification of this method is its clipping limit which is acquainted with defeat the clamor heightening issue. The author of [22],[23] illustrates that the histogram of an image is clipped by a pre-accepted value before it calculates Cumulative Distribution Function (CDF) and Block Size (BS) which is CLAHE's main destined and its Clipping Limit (CL) are predominantly used for the enhancement of low-contrast images [16].

$$
\operatorname{Img}(i)=I m g_{\min }+\sqrt{2 \alpha^{2} \ln \left(\frac{1}{1-P I C_{\text {input }}(i)}\right)}
$$

where $I m g_{\text {min }}$ is the lower bound of the pixel, $P I C_{\text {input }}(i)$ as transfer role, and $\alpha \square$ for scaling parameter in Rayleigh distribution with fixed to 0.04. Individual intensity value expressed for $\operatorname{Img}(i) \geq \operatorname{Img} g_{\min }$

$$
p(\operatorname{Img}(i))=\left(\frac{\operatorname{Img}(i)-I m g_{\min }}{\alpha^{2}}\right) \times \exp \left(\frac{\left(I m g(i)-I m g_{\min }\right)^{2}}{2 \alpha^{2}}\right)
$$

where $\alpha$ plays a significant role and will effect on the enhancement of image contrast. Contrariwise, increasing the value of $\alpha$ also intensified the noise levels. Furthermore, by linear contrast stretching the values is re-scaled by and illustrate as,

$$
\operatorname{Img}(\boldsymbol{i})=\left(\frac{\text { Frame }(\mathrm{i})-\text { Frame }_{\min }}{\text { Frame }_{\max }-\text { Frame }_{\min }}\right)
$$

where Frame $(i)$ transformation process value, Frame max $_{\text {and }}$ arame Fin $_{\text {min }}$ the maximum and minimum for the transformation process by utilizing a bi-straight introduction between four distinct mappings in graylevel sub-matrix to annihilate the limitation.

For the investigation, we used a $2 \mathrm{mp}$ IR night vision enable camera where the IR cut filter is covered to to get continuously night vision images. And we also tried different $850 \mathrm{~nm}$ IR illuminator sources like general IR and also IR led array along with the Matlab 2017a scietific tools.

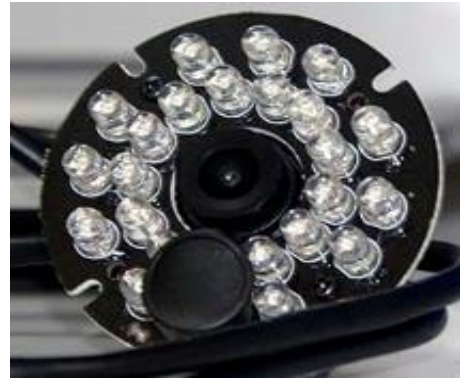

(a)

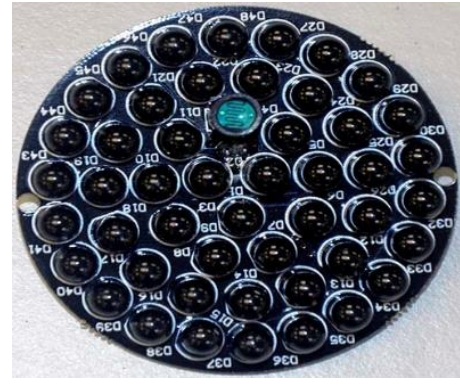

(b)

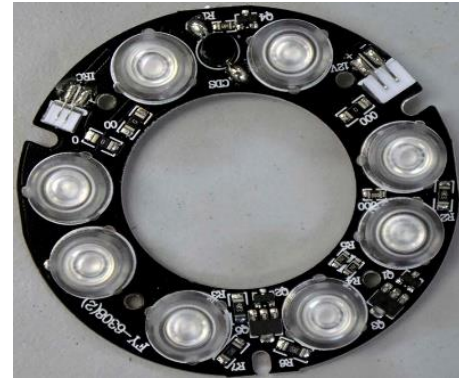

(c)

Figure 3. (a) $2 \mathrm{mp}$ IR Camera where the Black cap is enableing night mode and (b) \& (c) $850 \mathrm{~nm}$ NIR Illuminator

As found for a single frame the results has been shown in the Figure 4, where we particularly used a still image to for our experiment and it is shown blurred acquired reference image (Figure 4(a)) and followed by the background detection and ROI selection by some image processing thresholding techniques in the image (Figure 4(b)) which is require to adopt the best information from the image. Furthermore contrast enhancement technique CLAHE helped to provide clear veins for visualization can be seen as the acquired image (Figure 4(c)). 


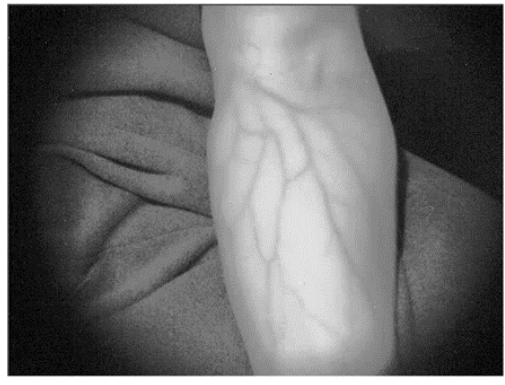

(a)

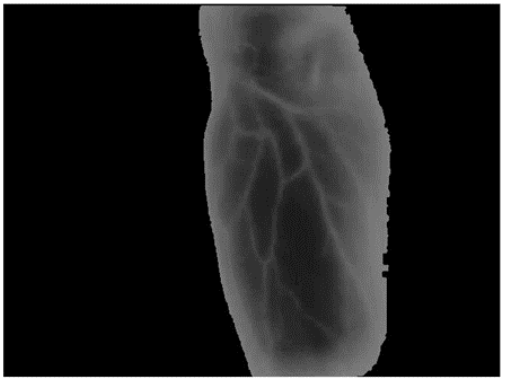

(b)

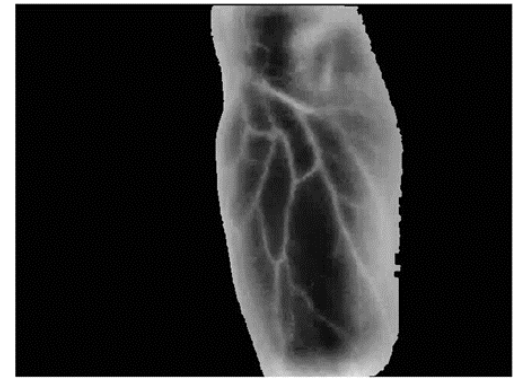

(c)

Figure 4. Result on a single image (a) Acquired Image (b) Threshhold image with binarizing and subtracting (c) Post-Enhanced image using CLAHE

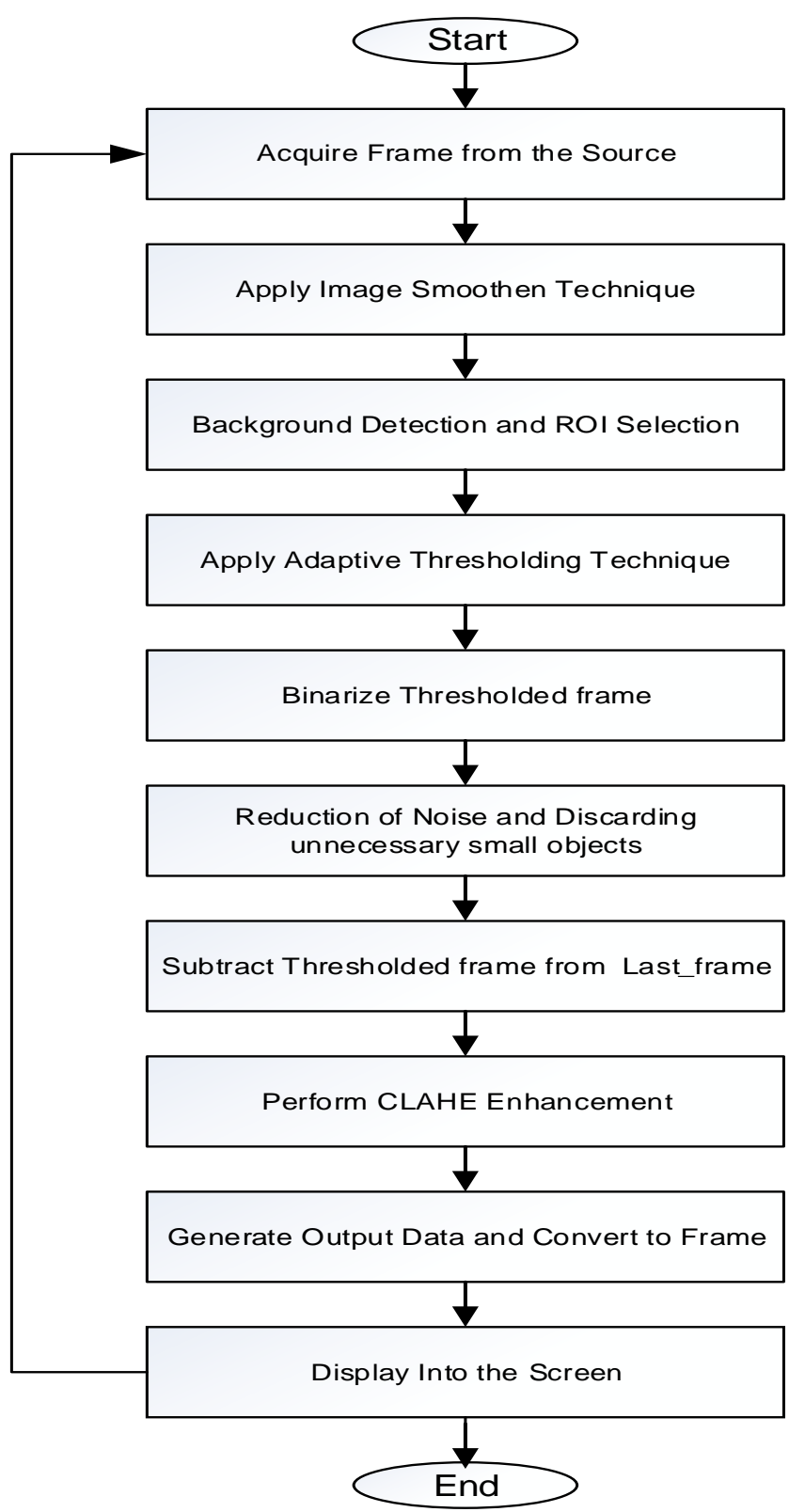

Figure 5. Block diagram of vein detection methodology 
These process start with the acquisition of vein frame from milieu and after receiving each of the frame, the system will automatically select the Region of Interest (ROI) location by thresholding function and enhance each of the frames using median filter. The step followed to the enhancement step the vein will automatically binarize that frame and subtract it from the frame which was received in the beginning and adaptive thresholding will be utilized to create a frame of each fragmented digits produce dark vein by changing the qualities in the state of values $<0.5$ will be 0 for the determination of the veins. CLAHE is an image processing technique which will provide high contrast image of the processed frame. After completion of all the step, the system will use CLAHE for the as Post Enhancement of the frame to provide brightest output of the detected veins. Finally, from the output data will be converted to a frame and and on the display it will be available to view.

\section{RESULTS AND ANALYSIS}

This section is divided into two parts. Th first part is for the video processing while the second part is to find out the effectiveness of the system in real-time. Some initial results reported in [24].

\subsection{Processing a Vein video File}

In this section, the continuous processing for each frame in a video sequence, each frame can be seen with very clean vein. So, phlebotomy can easily identify the vein as like the figure (Figure 6(a) - (b)).

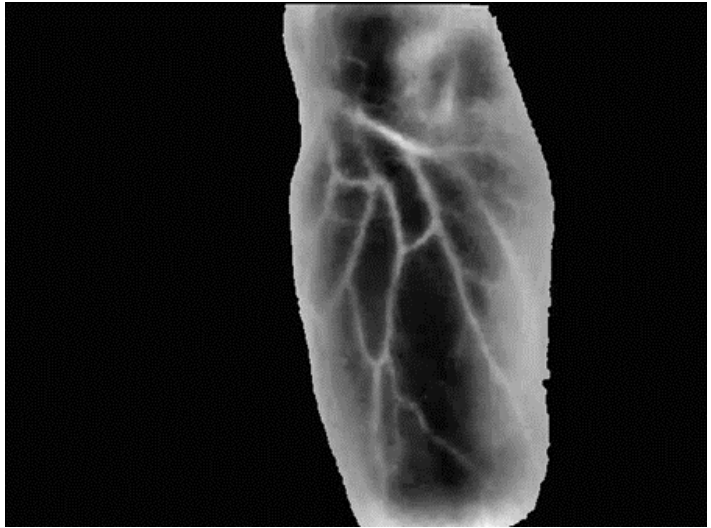

(a)

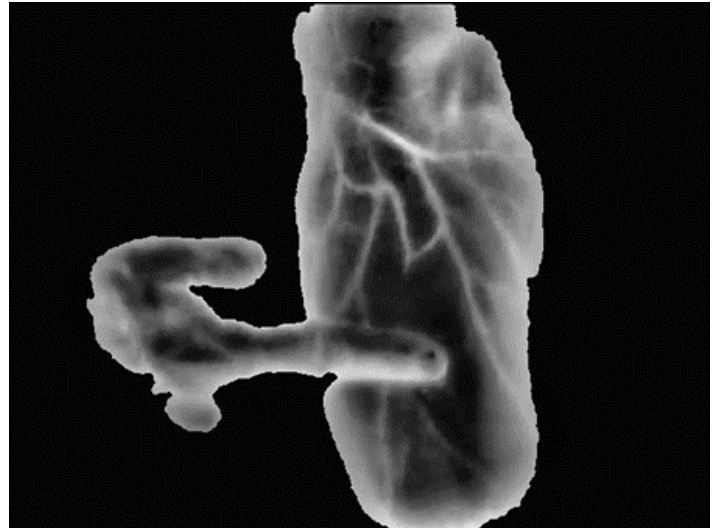

(b)

Figure 6. The resultant frames from video sequence (a) Frame 20, (b) Frame 100 displayed consecutively from a video file

\subsection{Processing in Real Time}

As the methodology stated, we take more sample from the real milieu and found the same result as for the video processing. In this scenario it is also considered that there was a $0.3 \mathrm{~ms}$ delay in between frame processing but still it can be considerable as though the frame rate was $15 \mathrm{fps}$.

As a final point, it can be seen from above that the procedure is going completely towards the solution of our objective to utilize the time in an efficient way and reduce the number of error in needle infusion in human body using the computational power to determine clearer vein. 


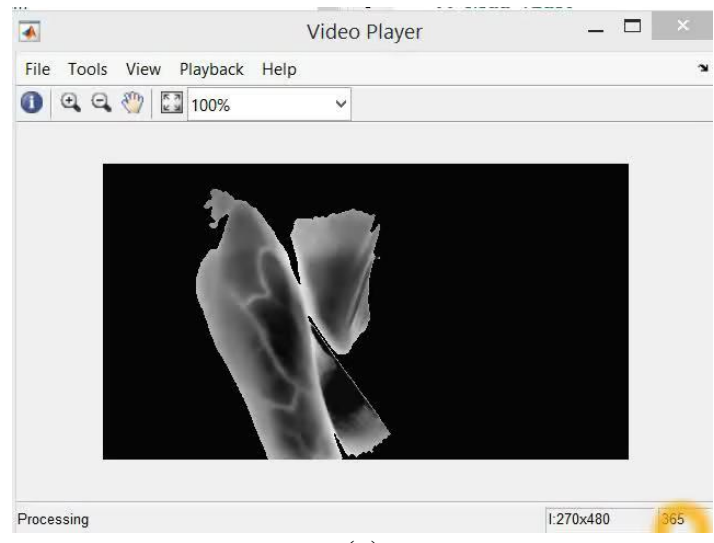

(a)
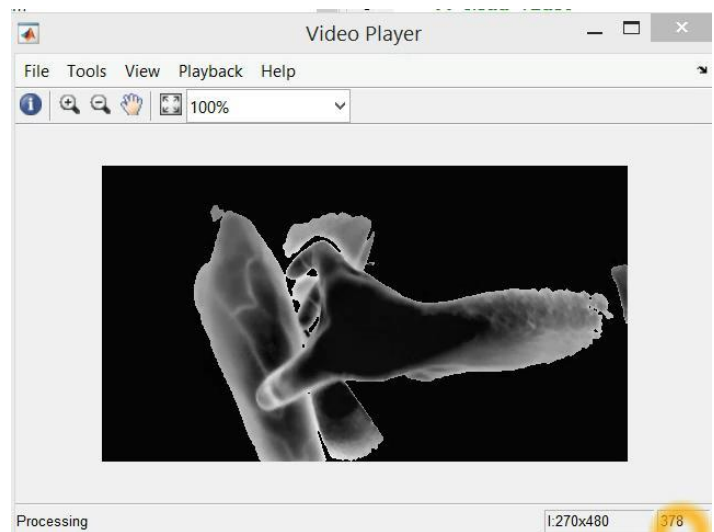

(c)
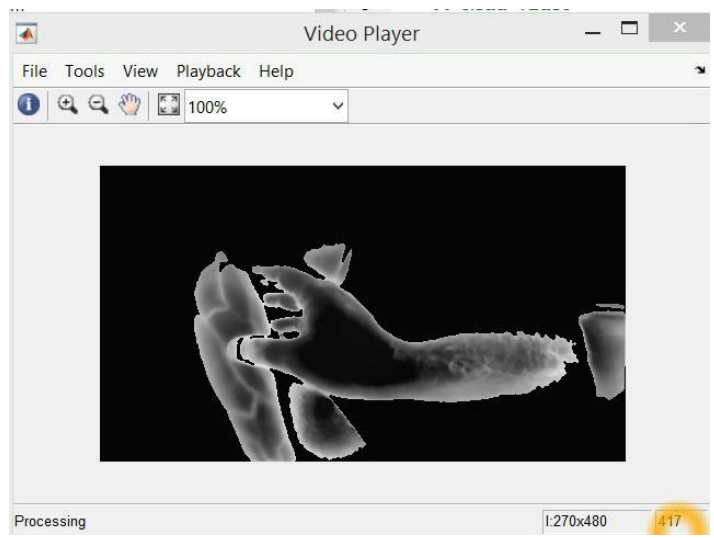

(e)

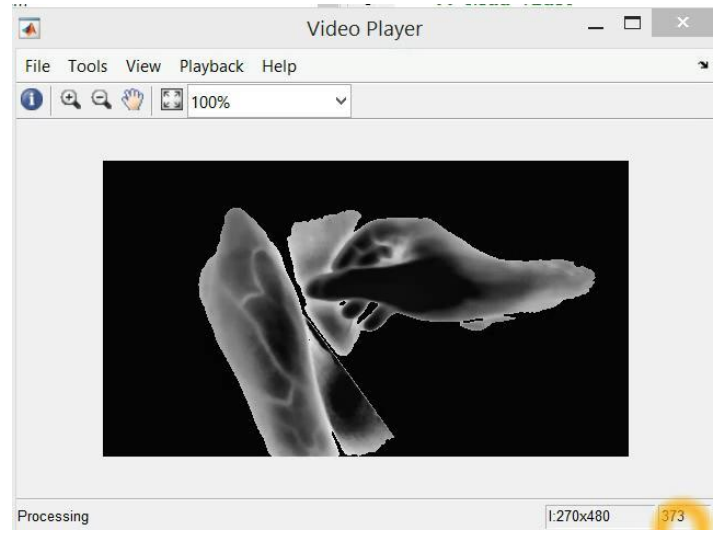

(b)

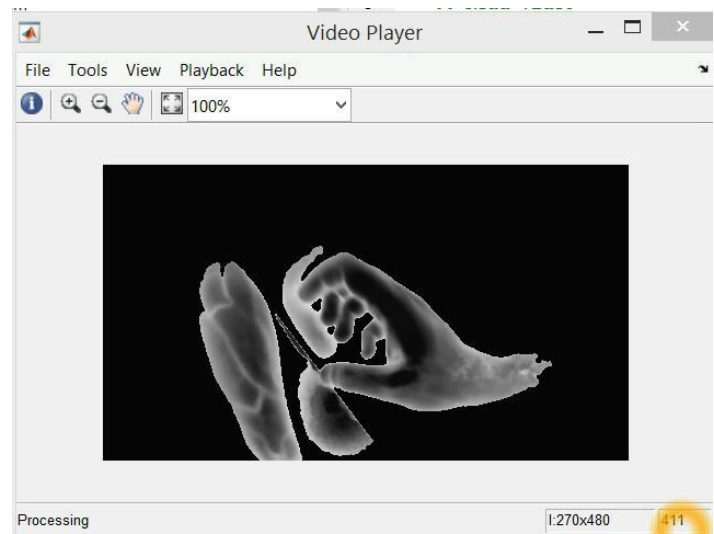

(d)

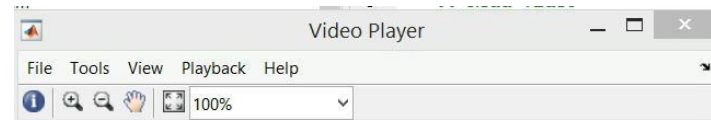

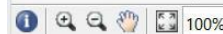

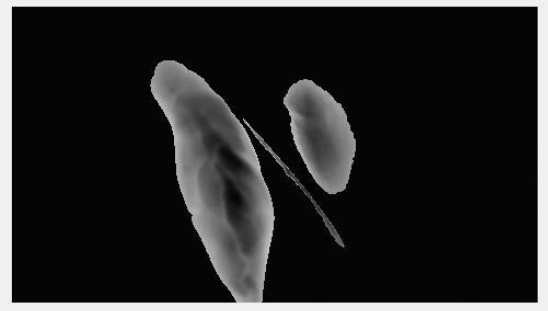

(f)

Figure 7. The resultant frames are captured from real-time by Microsoft Expression Encoder

\section{CONCLUSION}

This vein detection System is not a breakthrough in the medical imaging but still, it has a greater impact in daily medical practices which focus on the least number of calamity in needle infusion. The imminent of this process will focus on the performance and accuracy as a prospect along with in the most widely used handheld device Smartphone as the number of total smartphone user has crossed over 2 billion and its camera where the app will be considered more viable alternative because it will remove the cost of manufacturing or be pursuing new hardware system. So we are trying to spark the camera lenses manufacturer discernments to bring IR non-blocking camera into a smartphone. 


\section{REFERENCES}

[1] H. K. Al Ghozali, et al., "Vein detection system using infrared camera," 2016 Int. Electron. Symp., pp. 122-127, 2016.

[2] M. Kavya, "Vein Pattern Extraction : A Review," Int. J. Eng. Res. Technol., vol/issue: 6(5), pp. 869-870, 2017.

[3] M. Marathe, et al., "A novel wireless vein finder," Proc. Int. Conf. Circuits, Commun. Control Comput. I4C 2014, pp. 277-280, 2014.

[4] S. I. Shchukin, "Peripheral vein detection using electrical impedance method," J Electr Bioimp, vol. 8, pp. 79-83, 2017.

[5] B. Besra, "Extraction of Segmented Vein Patterns using Repeated Line Tracking Algorithm," in 2017 IEEE 3rd International Conference on Sensing, Signal Processing and Security (ICSSS) Extraction, pp. 89-92, 2017.

[6] N. S. Gnee, "A study of hand vein, neck vein and arm vein extraction for authentication," ICICS 2009 - Conf. Proc. 7th Int. Conf. Information, Commun. Signal Process., pp. 5-8, 2009.

[7] G. C. Meng, et al., "Prototype design for wearable veins localization system using near infrared imaging technique," 2015 IEEE 11th Int. Colloq. Signal Process. Its Appl., pp. 112-115, 2015.

[8] S. Yusoff, et al., "Review on Vein Enhancement Methods for Biometric System," Int. J. Res. Eng. Technol., vol/issue: 4(4), pp. 833-841, 2015.

[9] S. Crisan and B. Tebrean, "Low cost, high quality vein pattern recognition device with liveness Detection. Workflow and implementations," Meas. J. Int. Meas. Confed., vol. 108, pp. 207-216, 2017.

[10] R. R. Fletcher, et al., "Development of mobile-based hand vein biometrics for global health patient identification," Proc. 4th IEEE Glob. Humanit. Technol. Conf. GHTC 2014, pp. 541-547, 2014.

[11] M. Wadhwani, et al., "Vein Detection System using Infrared Light," Int. J. Sci. Eng. Res., vol/issue: 6(12), pp. 780-786, 2015.

[12] Y. Kanzawa, et al., "Human Skin Detection by Visible and Near-Infrared Imaging," IAPR Conf. Mach. Vis., pp. 503-507, 2011.

[13] S. Kacmaz, et al., "The Use of Infrared Thermal Imaging in the Diagnosis of Deep Vein Thrombosis," Infrared Phys. Technol., 2017.

[14] B. W. Ficke, et al., "Near-Infrared Vein Visualization in Index Finger Pollicization," J. Hand Surg. Am., vol/issue: 42(6), pp. 481.e1-481.e2, 2016.

[15] X. Dai, et al., "A fast vein display device based on the camera-projector system," IST 2013 - 2013 IEEE Int. Conf. Imaging Syst. Tech. Proc., pp. 146-149, 2013.

[16] J. Ma, et al., "Contrast Limited Adaptive Histogram Equalization Based Fusion for Underwater Image Enhancement," Preprints, pp. 1-27, 2017.

[17] R. Khan, "Comparison and Analysis of Various Histogram Equalization Techniques," Int. J. Eng. Sci. Technol., vol/issue: 4(4), pp. 1787-1792, 2012.

[18] M. Aarthy and P. Sumathy, "A Comparison of Histogram Equalization Method and Histogram Expansion," Int. J. Comput. Sci. Mob. Appl., vol/issue: 2(3), pp. 25-34, 2014.

[19] K. I. Ahmed, et al., "Enhanced Vein Detection from Video Sequences," Indones. J. Electr. Eng. Comput. Sci., vol/issue: 8(2), pp. 420-427, 2017.

[20] D. NV, "Satellite Imagery," $2016 . \quad$ [Online]. https://forum.nasaspaceflight.com/index.php?topic=39617.0.

[21] T. Jintasuttisak and S. Intajag, "Color retinal image enhancement by Rayleigh contrast-limited adaptive histogram equalization," in International Conference on Control, Automation and Systems, pp. 692-697, 2014.

[22] K. Zuiderveld, “Contrast Limited Adaptive Histogram Equalization,” Academic Press, Inc., 1994.

[23] J. Dash and N. Bhoi, "Detection of Retinal Blood Vessels from Ophthalmoscope Images Using Morphological Approach," ELCVIA, vol/issue: 16(1), pp. 1-14, 2017.

[24] K. I. Ahmed, et al., "Enhanced Vision Based Vein Detection System," in 4th IEEE International Conference on Smart Instrumentation, Measurement and Applications (ICSIMA), Putrajaya, Malaysia, 2017. 